EPJ manuscript No.

(will be inserted by the editor)

\title{
Residual conductance of correlated one-dimensional nanosystems: A numerical approach
}

\author{
Rafael A. Molina ${ }^{1}$, Peter Schmitteckert ${ }^{2}$, Dietmar Weinmann ${ }^{3}$, Rodolfo A. Jalabert ${ }^{3}$, Gert-Ludwig Ingold ${ }^{4}$, and \\ Jean-Louis Pichard ${ }^{1,5}$ \\ ${ }^{1}$ CEA/DSM, Service de Physique de l'Etat Condensé, Centre d'Etudes de Saclay, 91191 Gif-sur-Yvette, France \\ ${ }^{2}$ Institut für Theorie der Kondensierten Materie, Universität Karlsruhe, 76128 Karlsruhe, Germany \\ ${ }^{3}$ Institut de Physique et Chimie des Matériaux de Strasbourg, UMR 7504 (CNRS-ULP), 23 rue du Loess, BP 43, 67034 \\ Strasbourg Cedex 2, France \\ ' 4 Institut für Physik, Universität Augsburg, Universitätsstraße 1, 86135 Augsburg, Germany \\ ${ }^{5}$ Laboratoire de Physique Théorique et Modélisation, Université de Cergy-Pontoise, 95031 Cergy-Pontoise Cedex, France
}

October 31,2018

\begin{abstract}
We study a method to determine the residual conductance of a correlated system by means of the ground-state properties of a large ring composed of the system itself and a long non-interacting lead. The transmission probability through the interacting region and thus its residual conductance is deduced from the persistent current induced by a flux threading the ring. Density Matrix Renormalization Group techniques are employed to obtain numerical results for one-dimensional systems of interacting spinless fermions. As the flux dependence of the persistent current for such a system demonstrates, the interacting system coupled to an infinite non-interacting lead behaves as a non-interacting scatterer, but with an interaction dependent elastic transmission coefficient. The scaling to large lead sizes is discussed in detail as it constitutes a crucial step in determining the conductance. Furthermore, the method, which so far had been used at half filling, is extended to arbitrary filling and also applied to disordered interacting systems, where it is found that repulsive interaction can favor transport.
\end{abstract}

PACS. 73.23.-b Electronic transport in mesoscopic systems - 71.10.-w Theories and models of manyelectron systems - 05.60.Gg Quantum transport - 73.63.Nm Quantum wires

\section{Introduction}

Large experimental activities have recently been devoted to the study of the conductance of low-dimensional nanosystems like molecules, atomic chains, nanotubes, and quantum wires [1,2,3,4,5] with sizes typically of the order of the electronic Fermi wavelength. Since the screen' ing of the Coulomb interaction in such systems is less effective than in three dimensions, electronic correlations can no longer be neglected with respect to kinetic effects. In some of the systems mentioned, the Luttinger liquid behavior [6] 7] is relevant and might influence the transport properties.

The correlations become particularly relevant for low temperature electronic transport properties like the residual conductance and the interpretation of the experimental data requires a good understanding of transport through a region with strong correlations. However, this turns out to be a demanding task and various attempts have been made in this direction 8 [9].

The purpose of the present work is to contribute to the fundamental problem of transport through correlated nanostructures by studying a novel approach where the conductance is obtained from thermodynamic properties of a ring consisting of the nanosystem and a long lead. Such an embedding method has been actively pursued in the last few years [10, 11, 12,13, 14, 15, 16]. Here, we critically study its hypotheses and consequences in order to put it on a firm theoretical basis.

A powerful concept which was used for studying coherent transport through non-interacting systems is the Landauer-Büttiker formalism [18 19 which formulates a scattering problem between electron reservoirs. Although the electrons in the reservoirs interact, their density is very high such that the Coulomb energy to kinetic energy is small and they can be replaced by non-interacting quasiparticles. Hence, the reservoirs are well described by a Fermi distribution characterized by a temperature and a chemical potential. Within the scattering approach, the dimensionless residual conductance $g$ (in units of $e^{2} / h$ ) is given by the elastic transmission probability $\left|t\left(E_{\mathrm{F}}\right)\right|^{2}$ at the Fermi energy $E_{\mathrm{F}}$.

The situation becomes more complicated if electronelectron interaction is present in the scattering region because the passage of electrons may lead to the creation of excitations. However, for temperatures smaller than the 


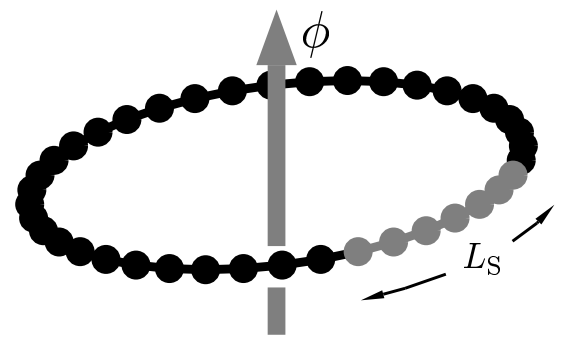

Fig. 1. The system considered within the embedding approach is a one-dimensional ring consisting of an interacting region (grey) of length $L_{\mathrm{S}}$ and a non-interacting lead (black) of length $L_{\mathrm{L}}$. The ring is threaded by an Aharonov-Bohm flux $\phi$.

characteristic excitation energy of the nanosystem, the idea of the Landauer-Büttiker formalism still applies [8] because all accessible states in the reservoir with an energy lower than the excitation energy are occupied. Inelastic processes are then forbidden. On the other hand, it remains non-trivial to determine the elastic transmission probability through a correlated system. Green function methods, while being conceptually adequate, require knowledge of the excited states and may become numerically quite involved.

An alternative approach consists in considering the ground state properties of a ring formed by the system of interest, which we will refer to as correlated system or nanosystem, and a very long non-interacting lead as depicted in Fig. 1. Within this embedding method, the relevant information about the conductance can be extracted by means of a flux threading the ring, which gives rise to a flux dependence of the ground-state energy and thus to a persistent current. This setup accounts for two important physical ingredients of coherent transport. First, the flux dependence of the ground-state energy provides information about extended states in the interacting region. Second, the two contacts between system and lead allow to transfer electrons into the system. This is an essential point in the description of conductance [20, which is not present when the persistent current is calculated for a correlated system without auxiliary lead.

Favand and Mila used the above described approach to compare, within a model of spinless fermions, the tunneling conductance of molecules with a Mott-Hubbard gap and of molecules with a dimerization gap [10. Sushkov used the same idea for a study of the $0.7 e^{2} / h$ anomaly observed in quantum point contacts 11,21. However, an important difference with respect to Ref. [10] is that he kept the interaction in the leads within the Hartree-Fock approximation. As the present authors have emphasized 12 , the extrapolation to infinite lead length can only yield meaningful results if no interaction is present in the auxiliary lead. Other important aspects discussed in Ref. 12 are the relevance of the contacts, the oscillation of the conductance as a function of the number of sites in the interacting region, and the role of static disorder. Meden and Schollwöck compared the results obtained within this approach to those of a perturbative functional renormal- ization group and showed that both give the same results at small values of the interaction strength, verifying scaling laws associated with Luttinger liquid behavior [13.14. Rejec and Ramšak tested the method, comparing its prediction with previous results for transport through single and double quantum dots. They presented a generalization to systems without time-reversal symmetry, using as an example a nanosystem which itself forms an AharonovBohm ring 15.16.

An approach related to the embedding method has recently been proposed by Chiappe and Vergés [17 in which the nanosystem and a small part of the leads are diagonalized exactly. In a second step, this subsystem is attached to semi-infinite leads and Green functions are employed to numerically calculate the conductance. The conductance through a one-dimensional interacting spin-system coupled to non-interacting leads was also studied by Louis and Gros by means of a Monte-Carlo based method [22].

The relationship between the conductance and the persistent current of a large ring has only been proven for non-interacting scattering systems. No rigorous proof has so far been put forward once electronic correlations are present in the scattering region. However, the conductance obtained by means of the embedding method satisfies all basic requirements and reproduces the correct behavior in various limiting cases. Moreover, in this work we demonstrate numerically for the one-dimensional case that in the limit of very large ring size, the effect of an interacting scatterer on the persistent current can be described by the amplitude of a transmission probability characterizing a $2 \times 2$ transfer matrix. Thus, transport through an interacting region can be understood as a non-interacting scatterer with interaction dependent parameters.

The remainder of the paper is organized as follows. In Section 2 we use Density Matrix Renormalization Group (DMRG) techniques to calculate the flux dependence of the persistent current through a ring composed of an interacting region and a non-interacting auxiliary lead in the limit where the latter becomes very long. It is found that this flux dependence reproduces the one expected for a non-interacting ring of equal length interrupted by a scatterer which can be characterized by a transfer matrix.

In the absence of Luttinger-like correlations in the ring it is meaningful to consider the limit of a very long auxiliary lead. In Section 3 we will explain how the extrapolation to infinite circumference can be performed in order to extract the interaction-dependent transmission coefficient and thus the conductance. For this scaling analysis, we make use of the charge stiffness instead of the persistent current, because it provides us with the same information but requires less numerical effort. Specific attention will be paid to the case of resonances, which appear when the coupling between system and leads is small and where the extrapolation has to be done with particular care.

In the literature, the embedding method has so far been discussed only for the case of half filling. In Section 4 we will present an extension to arbitrary filling. The important point is to choose the appropriate compensating background potential which ensures the correct 
charge density in the system even in the presence of interactions. While at half filling, it is straightforward to define the compensating potential from particle-hole symmetry, a self-consistent procedure is required away from half filling. In Section [5] we employ this new method to demonstrate that strong repulsive interactions can favor zero-temperature transport through strongly disordered systems.

We present our conclusions and perspectives in Section [6] In Appendix $\mathrm{A}$ we address the flux dependence of the ground state for a ring containing a local noninteracting scatterer, and obtain the asymptotic values and the finite size corrections to the charge stiffness. In Appendix $B$ we extend the approach to superconducting nanosystems and verify that the known behavior resulting from Andreev scattering at the two extremities of a superconducting nanosystem is reproduced. This illustrates the validity of the studied embedding method in an extreme limit where an attractive electron-electron interaction has dramatic effects.

\section{Flux dependence of the persistent current for large rings with a small scattering region}

The aim of this section is to demonstrate that the transport properties of an interacting region can be described as a non-interacting scattering problem with interaction dependent parameters. We start by considering the setup shown in Fig. 10 which will be employed to study the transport properties of a one-dimensional system of length $L_{\mathrm{S}}$. This system may contain a scattering potential and, possibly, electron-electron interaction may be present there.

The system is contacted by the two ends of an auxiliary one-dimensional lead of length $L_{\mathrm{L}}$ so that a ring of total length $L=L_{\mathrm{S}}+L_{\mathrm{L}}$ is formed. From this setup, transmission properties of the system can only be deduced if Luttinger liquid correlations [23] in the one-dimensional ring are absent. Therefore it is crucial that in the auxiliary lead no electron-electron interaction may be present. Not only, this allows to avoid Luttinger liquid correlations, but the electrons of the combined ring form a Fermi liquid in the limit of infinite lead length. According to Sushkov, one can give a general argument for $1 \mathrm{~d}$ spinless fermions on a ring demonstrating that they form a Fermi liquid though interactions act in a region of the ring 24, as far as it remains finite while the non-interacting lead becomes infinite. This is corroborated by our numerical findings presented below.

Information about the transmission amplitude $\left|t\left(E_{\mathrm{F}}\right)\right|$ at the Fermi energy $E_{\mathrm{F}}$ can be obtained by means of a magnetic flux $\phi$ threading the ring. For convenience, we introduce the dimensionless flux $\Phi=2 \pi \phi / \phi_{0}$ where $\phi_{0}=h / e$ is the flux quantum. The many-body ground state energy $E_{0}$ of the ring will oscillate with period $\phi_{0}$ as a function of the flux. The magnetic flux threading the ring breaks the symmetry between left and right moving electrons and thus gives rise to a persistent current $J(\Phi)$, which at zero temperature is given by $J(\Phi)=-\partial E_{0} / \partial \phi$.
For non-interacting scatterers, the persistent current $J(\Phi)$ decreases like $1 / L$ for large circumference $L$ of the ring. The leading contribution is found to read [25]

$$
J(\Phi)=-\frac{e v_{\mathrm{F}}}{\pi L} \frac{\operatorname{Arccos}\left(\left|t\left(k_{\mathrm{F}}\right)\right| \cos (\Phi)\right)}{\sqrt{1-\left|t\left(k_{\mathrm{F}}\right)\right|^{2} \cos ^{2}(\Phi)}}\left|t\left(k_{\mathrm{F}}\right)\right| \sin (\Phi)
$$

for an odd number of particles and

$$
J(\Phi)=\frac{e v_{\mathrm{F}}}{\pi L} \frac{\operatorname{Arccos}\left(\left|t\left(k_{\mathrm{F}}\right)\right| \cos (\Phi-\pi)\right)}{\sqrt{1-\left|t\left(k_{\mathrm{F}}\right)\right|^{2} \cos ^{2}(\Phi)}}\left|t\left(k_{\mathrm{F}}\right)\right| \sin (\Phi)
$$

for the case of an even number of particles in the ring. By Arccos, we denote the principal branch of the inverse cosine function which takes values in the interval $[0, \pi]$. The derivation of these results is outlined in Appendix $\mathrm{A}$

The persistent currents (11) and (21) depend on the properties of the non-interacting scatterer only through its transmission probability $\left|t\left(E_{\mathrm{F}}\right)\right|^{2}$ at the Fermi energy. This important feature allows us to determine the transmission probability and thus the residual conductance of the system from the persistent current of the composed ring. The relation becomes particularly simple for $\Phi=\pi / 2$, where the transmission coefficient at the Fermi energy can be expressed as 26 10,11]

$$
\left|t\left(E_{\mathrm{F}}\right)\right|^{2}=\left(\frac{J(\pi / 2)}{J^{0}(\pi / 2)}\right)^{2} .
$$

Here, $J^{0}$ is the persistent current for a clean ring of length $L$.

We now turn to an interacting nanosystem and demonstrate numerically that, in the limit of an infinitely long lead, the flux dependence of the persistent current is of the same form as in the non-interacting case of Eqs. (11) and (2). The interaction thus enters the result only through the transmission coefficient $\left|t\left(E_{\mathrm{F}}, U\right)\right|^{2}$.

Specifically, we have performed direct numerical calculations of the persistent current for a tight-binding model with $N$ interacting spinless fermions on $L$ sites described by the Hamiltonian

$H=-t \sum_{i=1}^{L}\left(c_{i}^{\dagger} c_{i-1}+c_{i-1}^{\dagger} c_{i}\right)+\sum_{i=2}^{L_{\mathrm{S}}} U\left[n_{i}-V_{+}\right]\left[n_{i-1}-V_{+}\right]$.

The hopping amplitude $t$ between nearest neighbors will be set to 1 and thus defines our energy scale. $c_{i}\left(c_{i}^{\dagger}\right)$ is the annihilation (creation) operator at site $i, n_{i}=c_{i}^{\dagger} c_{i}$ is the number operator, and the flux enters through the boundary condition $c_{0}=\exp (\mathrm{i} \Phi) c_{L}$. The length scale is given by the lattice spacing and the interaction acts between nearest neighbors inside the sample (sites $i=1$ to $L_{\mathrm{S}}$ ), but vanishes in the lead. To avoid depletion of electrons in the sample due to the repulsive interaction, we introduce a compensating potential $V_{+}$that acts as a positive background charge and ensures the local charge neutrality. For a half-filled ring, the compensating potential is equal to the filling factor $\nu=N / L$. Thus,

$$
V_{+}\left(\nu=\frac{1}{2}\right)=\frac{1}{2}
$$




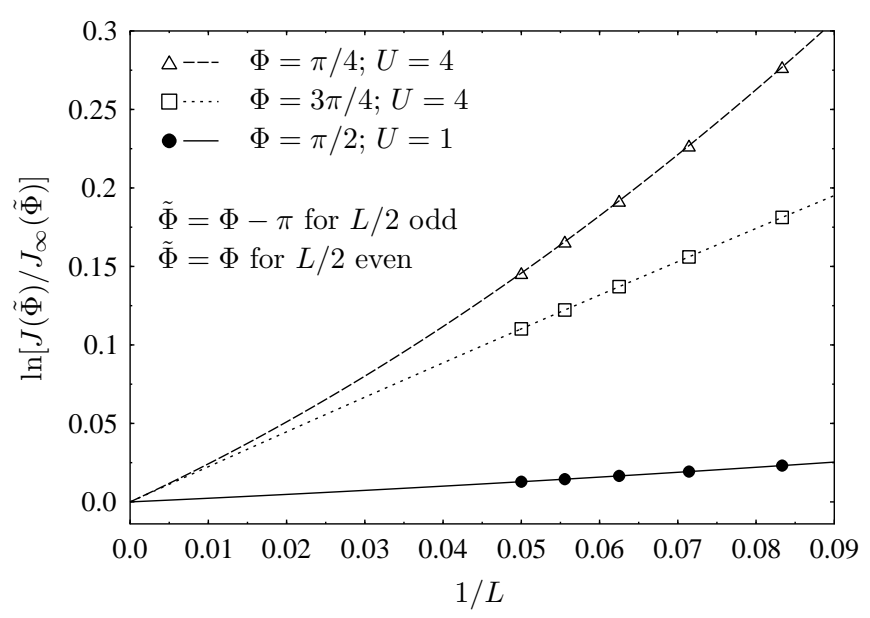

Fig. 2. The scaling of the persistent current with the total length $L$ is performed for a ring with system size $L_{\mathrm{S}}=6$ at half filling for several values of the flux $\Phi$ and the interaction strength $U$. The persistent current $J(\Phi)$ is depicted for even particle numbers $N=6,8,10$ while for odd particle numbers $N=7,9$, results for $J(\tilde{\Phi})$ with $\tilde{\Phi}=\Phi-\pi$ are shown. The extrapolation $L \rightarrow \infty$ has been performed by means of fits to second-order polynomials in $1 / L$.

guarantees particle-hole symmetry even in the presence of interactions. Outside half filling, this symmetry is broken and the compensating potential $V_{+}$becomes a function of $U, N, L_{\mathrm{S}}$ and $L_{\mathrm{L}}$ as we will discuss in Section 4

For the model (4) with system size $L_{\mathrm{S}}=6$ and at half filling, we have numerically determined the persistent current $J(\Phi)$ supported by the ground state for various values of $L_{\mathrm{L}}$ by means of a complex DMRG algorithm. With this implementation, we are able to treat not only the flux values $\Phi=0$ and $\Phi=\pi$ used in 12, where the Hamiltonian (4) can be represented by a real matrix, but also the general case of arbitrary flux where the matrix becomes complex. In order to determine the persistent current, we directly evaluate the current operator for the ground state, thereby avoiding the potentially difficult procedure of taking numerically the derivative of $E_{0}(\Phi)$.

The length dependence of the persistent current and the extrapolation to infinite lead length is shown in Fig. 2 for particle numbers $N=L / 2$ between 6 and 10. Motivated by the symmetry

$$
J(\Phi ; N \text { odd })=J(\Phi-\pi ; N \text { even })
$$

valid in the non-interacting case according to (11) and (2), we plot the interacting results corresponding to even and odd $N$ at flux values $\Phi$ and $\Phi-\pi$, respectively. As is shown in Appendix A, the scaling laws for even and odd $N$ may be different. However, making only the flux transformation of Eq. (6) allows us to obtain good asymptotic results from a single fit to the ensemble of data points for even and odd $N$. A second-order polynomial fit describes very well the deviation of the logarithm of the persistent current from its asymptotic value.

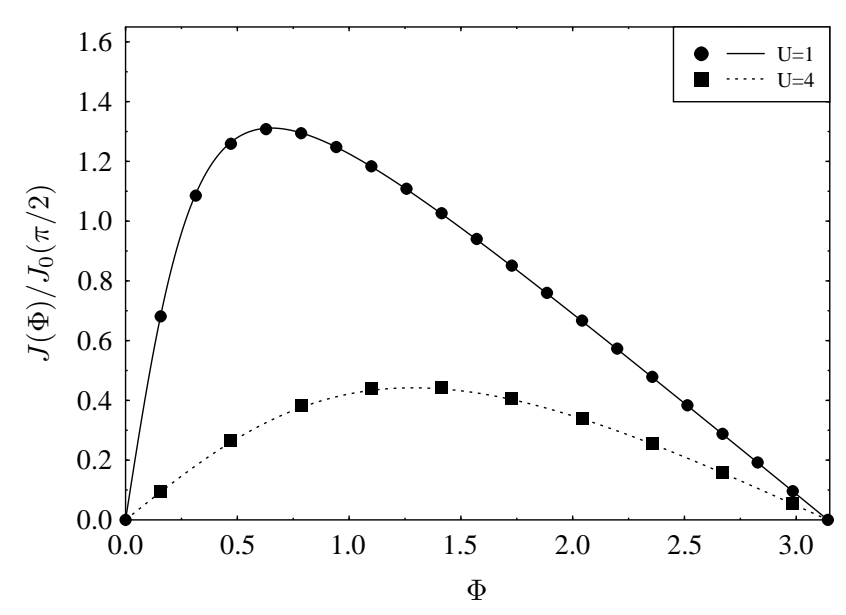

Fig. 3. The flux dependence of the persistent current for a system size $L_{\mathrm{S}}=6$ and half filling is shown for interaction strengths $U=1$ and 4 . The points represent DMRG results extrapolated to the limit of infinite leads (see Fig. 2). The lines represent the theoretical result (2) for a ring with a noninteracting scatterer and transmission amplitudes $|t|=0.938$ (solid line) and $|t|=0.425$ (dotted line).

The results presented in Fig. 2 indicate that the symmetry (6) holds even in the presence of electron-electron interaction and is independent of the interaction strength $U$. This provides numerical evidence that it should be possible to relate the persistent current in the presence of an interacting region to the persistent current for a noninteracting scattering problem.

The flux dependence of the persistent current $J(\Phi)$ for an even number of particles, extrapolated to the limit of an infinite lead, is presented in Fig. 3 for moderate and strong interaction, $U=1$ and $U=4$, respectively. At the filling factor $\nu=1 / 2$ used here, the interaction effects are expected to be most important. As can be seen from Fig. [3] the flux dependence of the persistent current is described very well by the expression (2) for the non-interacting case with transmission amplitudes of $|t|=0.938$ (solid line) and 0.425 (dotted line) for $U=1$ and 4 , respectively.

This demonstrates that, in the limit $L \rightarrow \infty$, the zerotemperature persistent current of a ring containing an interacting region is quantitatively described by the persistent current of a ring with a scatterering region. A single parameter, the interaction-dependent elastic transmission coefficient at the Fermi energy $\left|t\left(E_{\mathrm{F}}, U\right)\right|^{2}$ suffices to characterize the interacting sample, at least as far as the flux dependence of the ground state energy at zero temperature is concerned.

We emphasize that the DMRG technique employed here to calculate the persistent current of the ground state of the Hamiltonian (4) does not rely on any assumption. In particular, the DMRG technique does not require that the correlated nanosystem must be a Fermi liquid. But the fact that the expressions (11) and (2) for the persistent current hold in the infinite lead length limit even in the 
presence of an interacting region provides strong evidence that the Fermi liquid behavior is retained in this limit. This result is in agreement with the theoretical expectation mentioned above.

Our findings constitute a numerical "proof" that the extension of the relation between persistent current and transmission from a non-interacting to an interacting system is correct. Assuming that the composed ring forms a Fermi liquid, a discussion of the relation between the persistent current and the conductance had already been given in [16]. Together with the results of this section, this opens a road towards the calculation of the conductance for interacting nanosystems.

\section{Conductance from transmission for interacting scatterers}

Instead of the persistent current, we will, in the following, mostly work with the charge stiffness defined as:

$$
D=(-1)^{N} \frac{L}{2}(E(0)-E(\pi))
$$

which describes the change of the ground-state energy from periodic to antiperiodic boundary conditions. The factor $(-1)^{N}$ renders $D$ positive because the many-body ground state is diamagnetic for odd $N$ while it is paramagnetic for even $N$. This fact was proven by Leggett [27] for spinless fermions in the presence of arbitrary one-body potentials and arbitrary strength of electron-electron interactions. We prefer to work with the charge stiffness $D$ instead of the persistent current $J$ because it allows to avoid the use of a complex implementation of the DMRG algorithm and thus reduces the numerical effort.

For the case of a non-interacting scatterer, the flux dependence of the ground-state energy is derived in Appendix A ¿From Eqs. (25) and (28) it follows that for the limit of infinite lead length we have

$$
D=\frac{\hbar v_{\mathrm{F}}}{2}\left[\frac{\pi}{2}-\operatorname{Arccos}\left(\left|t\left(k_{F}\right)\right|\right)\right]
$$

independent of the parity of $N$. Solving (8) for the tunneling probability yields 12

$$
\left|t\left(k_{\mathrm{F}}\right)\right|=\sin \left(\frac{\pi}{2} \frac{D}{D^{0}}\right),
$$

where $D^{0}$ is the charge stiffness for a clean ring of length $L$ in the absence of electron-electron interactions. We note that for weak transmission $(|t| \ll 1), D$ is proportional to $|t|$.

We have verified that the transmission coefficients calculated from the stiffness using Eq. (9) as described in Ref. 12 coincide with the ones obtained by fitting the full flux dependence of the persistent current (Fig. 3) to a precision better than $0.5 \%$.

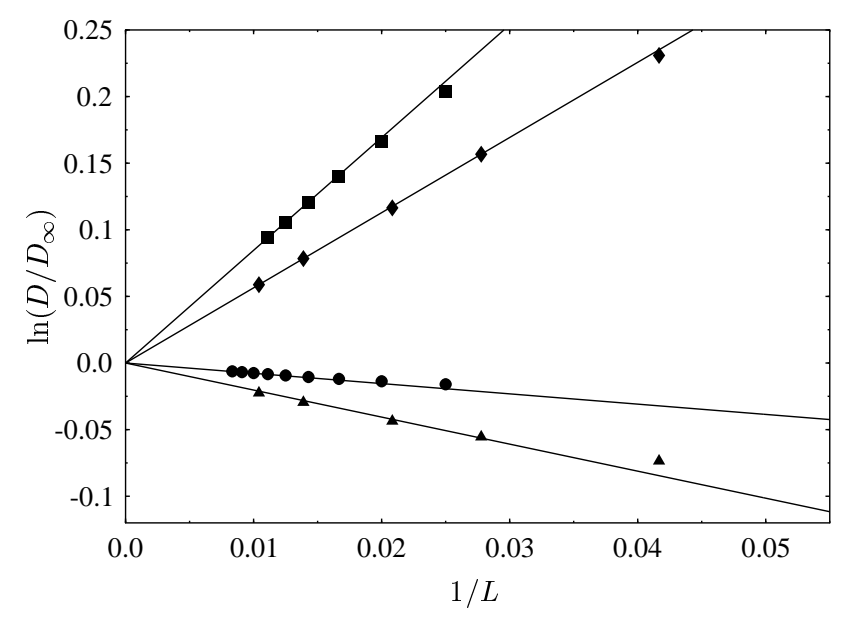

Fig. 4. The scaling of the logarithm of the charge stiffness with the ring size is shown for systems at half filling and $L_{\mathrm{S}}=20, U=3$ (squares), $L_{\mathrm{S}}=12, U=4$ (diamonds), $L_{\mathrm{S}}=17, U=1$ (circles), and $L_{\mathrm{S}}=13, U=2$ (triangles). The lines are linear fits to the large- $L$ behavior, providing the extrapolation to infinite ring size, i.e. $1 / L \rightarrow 0$.

\subsection{Scaling of the stiffness and extrapolation to infinite lead length}

As already discussed in Section 2 the limit of an infinitely long lead is required in order to obtain the conductance. While for the persistent current, we had been restricted to rather small ring sizes, the charge stiffness allows us to numerically treat rings almost an order of magnitude larger. This will enable us to take a closer look at the scaling of the charge stiffness with $1 / L$, even in difficult cases like in the presence of transmission resonances.

As the derivation of the charge stiffness as a function of the transmission amplitude in appendix A shows, the charge stiffness for large rings can be expanded in powers of $1 / L$. In the limit $L \rightarrow \infty$, a non-vanishing contribution given by (8) allows us to determine the conductance.

The leading corrections (26) and (29) for odd and even number of particles, respectively, are of order $1 / L$. Essential for the relevance of these corrections is their dependence on the derivatives with respect to $k$ of the transmission $|t|$ and the relative phase shift $\delta \alpha$ characterizing the scattering region. $\mathrm{d} \delta \alpha / \mathrm{d} k$ is proportional to the Wigner delay time [28. At resonances, the two derivatives may become very large. Then, only rings of circumference $L \gg \mathrm{d}|t| / \mathrm{d} k, \mathrm{~d} \delta \alpha / \mathrm{d} k$ allow to perform a reliable extrapolation to the asymptotic limit. This situation will be discussed in Section 3.2 Outside resonances, we found that the extrapolation can usually be performed with rings about three or four times as large as the scattering region.

In Fig. 4 we present the deviation of the logarithm of the charge stiffness from its asymptotic value as a function of the inverse circumference $L$ of the ring. This plot is the analogue of Fig. 2 where the scaling of the persistent current was depicted, but now the size of the nanosystem 
is up to a factor of three larger. In all cases shown here, we are far away from any resonance.

The scaling with the ring size has been described by different laws in the literature. A parabolic fit was assumed in the first paper of Favand and Mila [10] while a linear fit to the deviations of the logarithm was employed in our previous paper 12. Different polynomial scalings were compared by Meden and Schollwöck 13. In the present work, we have used a linear scaling for the deviations of $\ln (D)$. A second-order fit becomes necessary when numerical limitations prevent us from attaining sufficiently large ring sizes as it has been the case for the persistent current (cf. Fig. 2).

For the extrapolation of the charge stiffness in the cases presented in Fig. 4 it is sufficient to use the scaling law

$$
D\left(U, L_{\mathrm{S}}, L\right)=D_{\infty}\left(U, L_{\mathrm{S}}\right) \exp \left(\frac{C\left(U, L_{\mathrm{S}}\right)}{L}\right)
$$

to determine the asymptotic value $D_{\infty}\left(U, L_{\mathrm{S}}\right)$. The conductance is then obtained from (9) as

$$
g=\sin ^{2}\left(\frac{\pi}{2} \frac{D_{\infty}}{D^{0}}\right) .
$$

This procedure had been used in Ref. 12 to compute the influence of the interaction strength on the conductance of correlated nanosystems at half filling. The conductance of a clean system decreases with the interaction strength (see the solid line in Fig. 8) for even numbers of particles, and remains perfect $(g=1)$ for odd numbers of particles independently of the interaction strength.

\subsection{Scaling close to transmission resonances}

The leading correction (26) or 29) to the charge stiffness may play an important role close to transmission resonances, where the Wigner delay time and $\mathrm{d}|t| / \mathrm{d} k$ are large. We illustrate the difficulties in the extrapolation procedure present in this case by considering a nanosystem separated from the auxiliary lead by two tunnel barriers (cf. Fig. 5). In order to tune the Fermi energy of the ring to a resonance, we introduce an electrostatic potential $V_{0}$ between the tunnel barriers of height $V_{\mathrm{b}}=1$. A singleparticle term $V_{\mathrm{b}}\left(n_{1}+n_{L_{\mathrm{S}}}\right)+V_{0} \sum_{i=2}^{L_{\mathrm{S}}-1} n_{i}$ is thus added to the Hamiltonian (4). The electron-electron interaction is present on all $L_{\mathrm{S}}$ sites including the two barrier sites but the lead remains non-interacting as usual. We note that the additional potential $V_{0}$ will change the electron density in the nanosystem.

Resonances occur whenever the ground state energies of the ring with $N+1$ particles and $N$ particles inside the double-barrier system are identical. In the absence of electron-electron interaction, this implies that the energy of the first unoccupied single-particle state of the well between the barriers lines up with the Fermi energy of the leads. When the degeneracy between ground states with different number of particles in the system appears, the energetic cost for transporting a particle through the system is zero and the transmission is one.

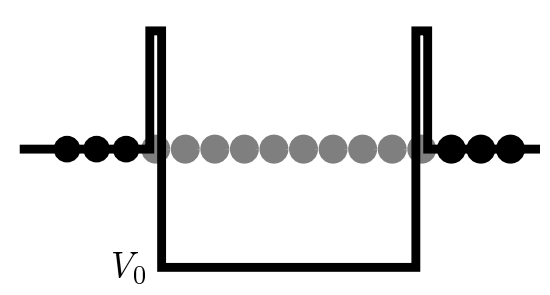

Fig. 5. Sketch of the site potentials used for the double barrier system. Electron-electron interaction is present only on the grey sites.

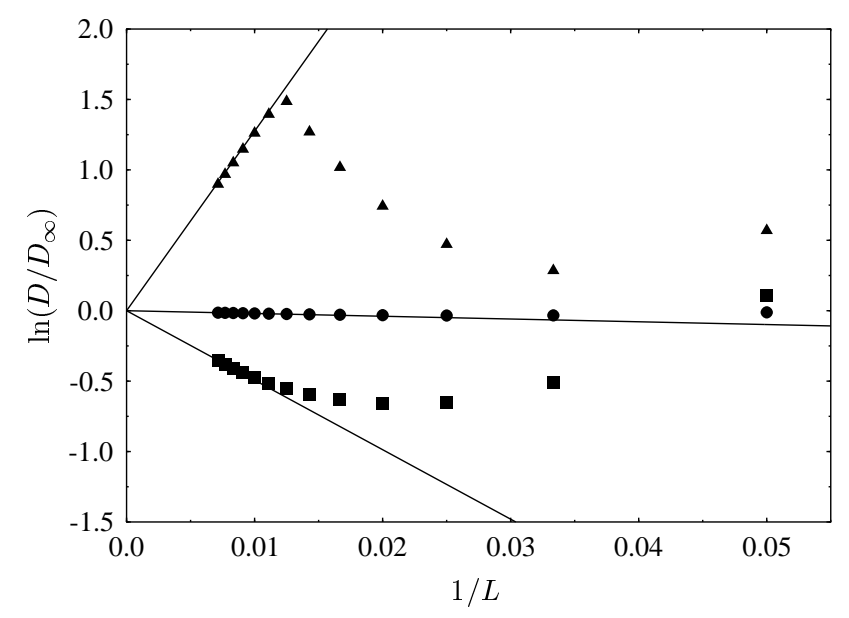

Fig. 6. Scaling towards the asymptotic value of the stiffness $D$ for a weakly coupled nanosystem with $L_{\mathrm{S}}=10$ and $U=1$. The circles, squares, and triangles correspond to electrostatic potentials $V_{0}=-0.8$ (out of resonance), $V_{0}=-1.4$ (just to the right of a resonance), and $V_{0}=-1.5$ (just to the left of a resonance), respectively.

For the reasons discussed in the previous section (see also Appendix (A), this case is characterized by a slow convergence towards the limit $L_{\mathrm{L}} \rightarrow \infty$. Large lead lengths are then needed because the very rapid changes of the transmission as a function of $k$ lead to large corrections. Another reason consists in the difficulty to maintain the resonance condition for the electron density of the nanosystem in the scaling procedure. However, even in this unfavorable case, the conductance can be obtained by going to larger systems and taking the asymptotic value with a greater care than for the non-resonant case.

Fig. 6] shows for the example of a double-barrier system how one can extrapolate to the asymptotic value of the stiffness in three cases, one favorable and two unfavorable. The ratio $\ln \left(D / D_{\infty}\right)$ is given as a function of the inverse total length of the ring. The circles correspond to $V_{0}=-0.8$ and $U=1$, situated in the valley between two resonances where the conductance is small. In this case, the extrapolation is straightforward and the slope is very small. The other two cases are different. Taking 
$V_{0}=-1.4$ and $U=1$ (depicted by squares), we are just to the right of a resonance. The corrections to the scaling formula (10) are very large for small ring sizes, and a naive extrapolation from there can give wrong values (even $g>1$ ) for the conductance. In order to test that the asymptotic value for $D$ is approached, one calculates the parameters $C$ and $D_{\infty}$ of the scaling formula (10) for two different values of $L$ and one continues to increase $L$ until the slope $C$ and the asymptotic stiffness $D_{\infty}$ converge to constant values. In the case $V_{0}=-1.5$ shown by triangles in Fig. 6. we have first determined $C$ and $D_{\infty}$ assuming the scaling law (10) for $L=30$ and $L=40$. Because the procedure gives different results when we take $L=40$ and $L=50$, we were forced to increase $L$. Since the values for $C$ and $D_{\infty}$ obtained with $L=120,130$ and 140 do not vary, we assume that one has reached the asymptotic regime. This procedure can require large values of the total length $L$ of the ring, which are difficult to reach for large filling factors $\nu$. Using a fit with more parameters can be an option when the convergence is slow, but the extrapolation must be done very carefully. The behavior of the stiffness $D$ as a function of the length $L$ in this last example is quite complicated because the density in the lead cannot be kept perfectly uniform and therefore the resonances move as a function of the increasing size of the ring. This extreme case illustrates the potential difficulties which must be solved in order to get reliable values for $g$ in the vicinity of transmission resonances from this method. For $V_{0}=-1.5$ (just to the left of a resonance), the slope has changed sign and we still need to go to big ring sizes for a reliable extrapolation.

In Fig. 7] we depict the results of the conductance, evaluated using the previous extrapolations for the twobarrier system. We compare the results for $U=0$ and $U=1$. The values for $U=0$ have been obtained in the same way as the values for $U=1$, using DMRG and the extrapolation. They are found to agree with results from a non-interacting Green function calculation. The fact that we do obtain perfect conductance $(g=1)$ at resonances supports our claim that the asymptotic procedure is capable of yielding the correct transport properties. In Fig. 7] we show the slope $C\left(U, L_{\mathrm{S}}\right)$ of the scaling law (10). As one can see, the resonance structure is clearly reflected by the slope of the scaling curves. The jumps in the slope coincide with the values for which the dimensionless conductance approaches its maximum value of one. The slope is closely related to the behavior of $\mathrm{d}|t| / \mathrm{d} k$. As expected, the interaction $U$ changes the position of the peaks and their widths.

\section{Conductance outside half filling}

As stated in the introduction, most of the applications of the embedding method have so far been restricted to half filling. In the previous section, we have maintained half filling in the average over the composed ring, but the filling of the correlated system itself depended on the potential $V_{0}$ between the barriers. As an even more general situation, we now consider the case where the filling in

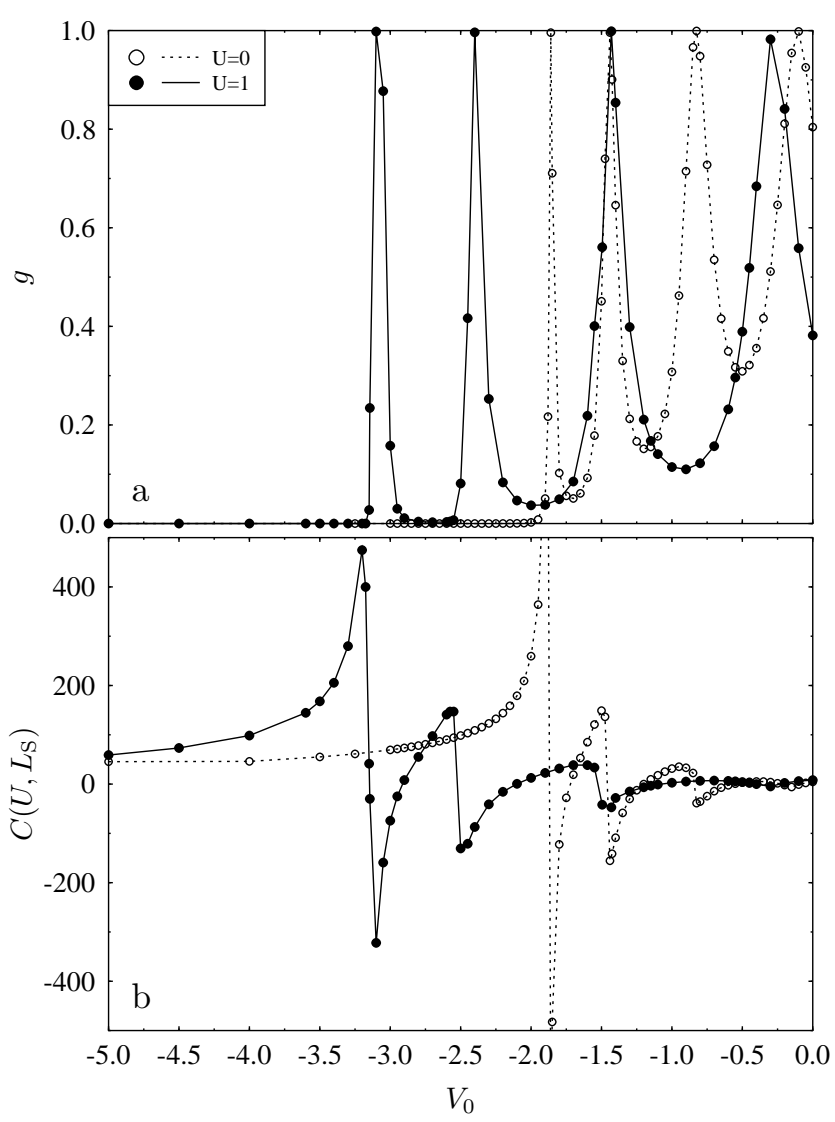

Fig. 7. (a) Conductance $g$ and (b) slope $C\left(U, L_{\mathrm{S}}\right)$ of the scaling law (10) are shown as a function of the electrostatic well potential $V_{0}$ for the configuration of Fig. 5 Results for interaction strength $U=1$ are indicated by full symbols and a solid line while the open symbols and the dotted line represent results for the non-interacting case $(U=0)$.

the composed ring has an arbitrary value $\nu$. The half-filled systems exhibit particle-hole symmetry, and therefore the compensating potential $V_{+}=1 / 2$ required to yield charge neutrality inside the nanosystem is known a priori. If we want to ensure a given constant filling $\nu$ for the nanosystem and the lead even when the lead length is changed, $V_{+}$ becomes a function of the interaction strength and the ring size. In this section, we extend the method to nanosystems outside half filling which are well coupled to the lead. By choosing the appropriate particle number, the same filling is imposed in the auxiliary lead in order to obtain the transmission coefficient $\left|t\left(E_{\mathrm{F}}, U\right)\right|^{2}$ at the corresponding Fermi energy and to ensure a better convergence towards the limit of infinite lead length.

In order to determine $V_{+}$for an arbitrary filling $\nu$, we begin with an initial guess for $V_{+}$and calculate numerically the corresponding number of particles contained inside the nanosystem. Then, we adjust $V_{+}$performing an iterative solution of the problem using the NewtonRaphston method. In principle, $V_{+}$depends on $U$ and $L_{\mathrm{L}}$. For example, for $L_{\mathrm{S}}=8, \nu=3 / 8$ and $U=3, V_{+}$varies 


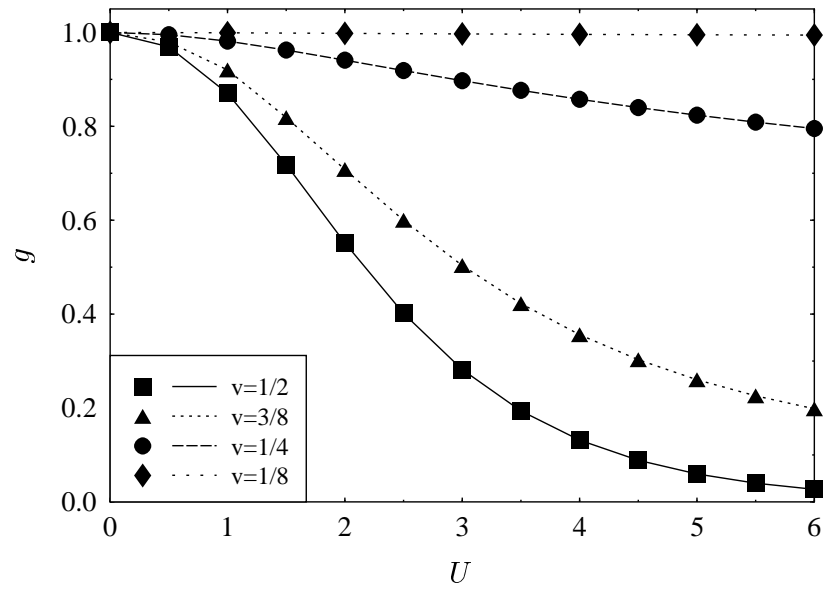

Fig. 8. Conductance as a function of $U$ for different filling factors $\nu$ of a correlated system of size $L_{\mathrm{S}}=8$.

from 0.1924 to 0.1939 as $L_{\mathrm{L}}$ is doubled from 24 to 48 . At a fixed interaction strength, the dependence of $V_{+}$on $L_{\mathrm{L}}$ becomes negligible beyond a certain $L_{\mathrm{L}}$, and can then be ignored. Therefore, the iterative procedure has only to be performed until a limiting value for $V_{+}$has been attained. Then this value can be kept for larger ring sizes from which $C$ and $D_{\infty}$ are determined, using the same scaling law as at half filling.

In Fig. 8, the conductance of a nanosystem of length $L_{\mathrm{S}}=8$ perfectly coupled to the lead is given as a function of the interaction strength $U$ at different filling factors $\nu$. Since the filling is kept uniform everywhere in the ring, the curves characterize $g\left(E_{\mathrm{F}}, U\right)$ at the corresponding Fermi energy $E_{\mathrm{F}}$. At $\nu=1 / 8$ (short dashed line), in average only one particle is left in the nanosystem. In the absence of other particles to interact with, the dimensionless conductance therefore equals one, independently of the interaction strength. For larger filling factors, the conductance $g$ decreases with increasing interaction strength $U$ and this decay becomes more pronounced as the filling factor is increased. The rather sharp drop of the conductance occurring at half filling around $U=2$ is a precursor of the Mott transition expected in the thermodynamic limit. The conductance above half filling can be obtained from $g(\nu)=g(1-\nu)$ as a consequence of particle-hole symmetry. The influence of the interaction strength on the conductance is thus the strongest at $\nu=1 / 2$ as expected.

\section{Conductance for disordered nanosystems}

Having demonstrated that the conductance of a correlated nanosystem can be obtained from the charge stiffness after embedding it into a large noninteracting ring, we now apply this method to the problem of interacting electrons in disordered systems. The effect of repulsive interactions in a disordered system is a controversial issue 29. It is often believed that interactions impede transport. This

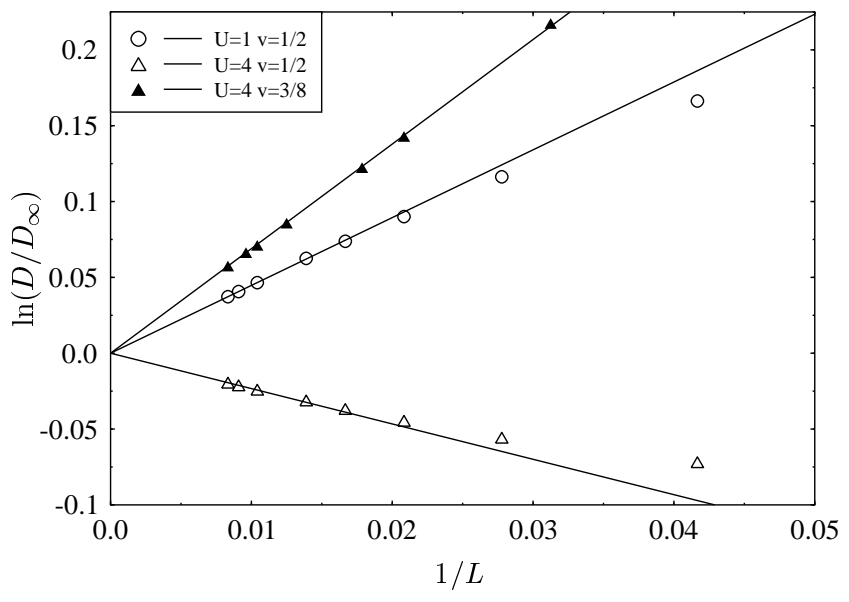

Fig. 9. Scaling towards the asymptotic value of the stiffness $D$ for disordered samples $(W=5)$. For the same disorder realization, two values of the interaction are shown for half filling. Open triangles represent $U=4$ and open circles the case $U=1$. The negative slope in the former case corresponds to a charge reorganization and the conductance $g=0.34$ for $U=4$ is greater as compared to $g=0.018$ for $U=1$. Results for the same disorder configuration with $U=4$ and $\nu=3 / 8$ are displayed with filled triangles. The conductance in this case is $g=0.0011$, demonstrating that the charge reorganization depends on the filling.

belief comes from perturbative arguments showing that interactions reduce the density of states at the Fermi level of a disordered metal [30] and open a gap for a strongly disordered insulator 31. On the other hand, in the strong disorder limit zero temperature transport can be enhanced by an interaction-induced delocalization of the many-body ground state. This was demonstrated for the special case of half filling in Ref. [12. In the following, we will study the role of the filling factor in the delocalization process.

We include the disorder potential into the Hamiltonian (4) by adding a term

$$
H_{\mathrm{dis}}=W \sum_{i=1}^{L_{\mathrm{S}}} v_{i} n_{i},
$$

where $W$ denotes the disorder strength, and the $v_{i}$ are independent random variables, equally distributed within the interval $[-1 / 2,1 / 2]$. The disorder potential is only present within the nanosystem of length $L_{\mathrm{S}}$.

We start by verifying that the scaling towards infinitely large rings also works in the presence of disorder. Fig. 9 depicts the dependence of the logarithm of the charge stiffness $D$ on the ring size $L$ for a sample with $W=5$ for interaction strengths $U=1$ (circles) and 4 (triangles). The disorder realization is the same in both cases. The open symbols refer to $\nu=1 / 2$ while the full symbols correspond to $\nu=3 / 8$. In all cases the scaling works well, and thus reliable values for the conductance can be extracted.

The analysis of individual samples helps us to understand the physical mechanisms involved when disorder 


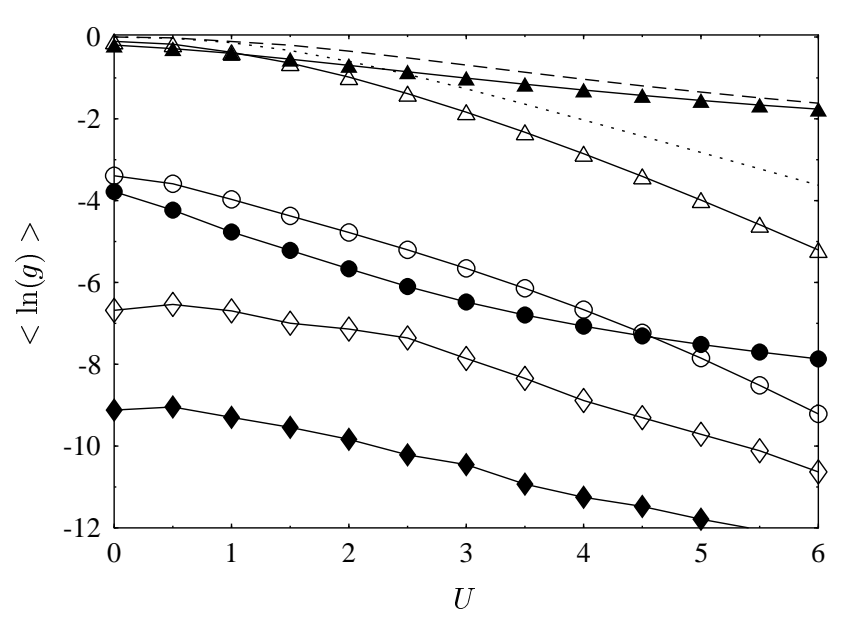

Fig. 10. Logarithmic ensemble average of the conductance as a function of the nearest neighbor repulsion $U$ for a disordered nanosystem of length $L_{\mathrm{S}}=8$. The open symbols correspond to half-filled nanosystems, and the filled symbols to a filling factor $\nu=3 / 8$. The triangles correspond to $W=1$, the circles to $W=5$ and the diamonds to $W=9$. For $W=0$, the dashed line corresponds to $\nu=3 / 8$ and the dotted line to $\nu=1 / 2$, respectively.

and interactions are both relevant 32,33. Studying the evolution of the ground state energy or the electron density as a function of $U$, we can detect charge reorganizations at critical values of the interaction strength. For the sample shown in Fig. [9 we have, at half filling, a charge reorganization in the ground state structure around $U=4$. Charge reorganizations appear when a ground state configuration which is well adapted to the non-interacting case, where the fermions are located in the minima of the disorder potential, changes towards a Wigner-like crystalline structure which is energetically favorable at strong repulsive interaction. This resonant situation increases the conductance at the particular (sample dependent) crossover value of the interaction. In other samples the charge reorganizations can occur at different values of the interaction or can even be absent, depending on the disorder realization. Reducing the filling makes the charge reorganizations less likely. For the charge reorganizations of the disordered case, we typically obtain a negative slope for the asymptotic scaling of $D$, similar to the case of clean systems with odd number of particles [12. In both cases, a degeneracy of different charge configurations in the nanosystem occurs.

In Fig. 10 the ensemble average of the logarithm of $g$ is given as a function of $U$ for disorder strengths $W=1$ (triangles), $W=5$ (circles), and $W=9$ (diamonds) and filling factors $\nu=1 / 2$ (open symbols) and $\nu=3 / 8$ (full symbols). One can see from the increase of the average conductance at weak interaction in the strongly disordered case, $W=9$, that the nearest neighbor interaction has stronger delocalization effects around half filling. The results for the clean case show Mott insulator like behavior at half filling (dotted line). The decay of the typical value of $g$ as a function of $U$ is faster for the half-filled case than for $\nu=3 / 8$. When we introduce a random potential in the nanosystem, the reduction of the typical conductance due to localization effects is more important outside half filling.

The larger the density, the better is the screening of the random potentials. In the case of weak disorder, $W=1$, this gives rise to a crossing of the curves with $\nu=1 / 2$ and $\nu=3 / 8$ as $U$ increases. For stronger disorder, this crossing occurs at larger values of $U(U=4.5$ for $W=5)$ and for very strong disorder $(W=9)$ the crossing cannot be observed in the figure.

We can also see in Fig. 10 that in the strong disorder case (here $W=9$ ), nearest neighbor interactions can favor transport. This enhancement of the typical elastic transmission, and hence of the zero temperature conductance, is maximal around $U=0.5$ and, though mainly characteristic for half filling, it persists outside $\nu=1 / 2$.

The charge reorganization induced by repulsive interactions in strongly disordered systems and its associated delocalization effect was first observed in the persistent current of nanosystems 32 33] forming a ring (without the auxiliary lead introduced within the embedding approach). As our results demonstrate, the same effects can be found in the conductance $g$. Considering a given nanosystem, one observes a similar resonance structure [12] as for the persistent current 32 33, although the individual peaks are wider for the conductance than for the persistent current.

\section{Summary}

The residual conductance of a correlated nanosystem can be obtained from the charge stiffness or from the persistent current of a ring composed of the system and an auxiliary non-interacting lead. Using DMRG for spinless fermions, we have numerically studied basic properties of this embedding approach. In particular, we have demonstrated that the flux dependence of the persistent current for an interacting system and a non-interacting lead agrees with the flux dependence of a non-interacting ring with a scatterer, in the limit of infinite lead length. This allows to extract the interaction dependent transmission coefficient of the interacting system, and hence its residual conductance.

A detailed analysis of the finite-size corrections has been performed for the charge stiffness. The main features of these corrections can be understood from the analysis of the non-interacting case. Away from transmission resonances, we obtain a very good scaling behavior already for not too large lead lengths, and the conductance of the correlated nanostructure can be readily obtained. Close to resonances the asymptotic limit of large lead lengths is problematic and only by considering very long leads we obtain the correct asymptotic behavior. Even in these special cases, the results for the conductance agree with our expectation for the resonant tunneling behavior in a double barrier structure. 
It is straightforward only at half filling to keep the electron density in the correlated system fixed when changing the ring size. We have demonstrated that an extension of the method to arbitrary filling factors is feasible provided the compensating potential is adjusted appropriately. For clean samples, it was observed that the decrease of the conductance with increasing interaction strength is strongest at half filling and becomes weaker as the filling factor changes towards smaller or larger values.

Another extension consists in the introduction of disorder in the correlated system. Charge reorganizations of the ground state appear at sample-dependent values of the interaction strength, affecting the long lead scaling and the asymptotic values. In the ensemble averages, we obtain for weak disorder a decreasing conductance as a function of the interaction strength. However, for strong disorder we have shown that a nearest neighbor repulsion can enhance the average of the logarithm of the conductance for spinless fermions in one-dimensional samples. This enhancement persists outside half filling, although it becomes weaker.

So far, the approach is still limited to spinless fermions and single-channel leads, although the system itself can be arbitrary. Nevertheless, the method is well suited to study the role of the contacts between the nanosystem and the leads. Furthermore, interesting phenomena like even-odd oscillations of the conductance with the number of fermions were found with this approach 12,34. In the absence of spin-flip scattering, the generalization to electrons with spin is straightforward. Indeed, first calculations for the Hubbard model have already been performed 12 . These and further issues will be explored in more detail in future work.

RAM wishes to thank J. Ségala for reminding him of some properties of the Chebyshev polynomials. We gratefully acknowledge financial support from the European Union through the RTN program (Contract No. HPRN-CT-2000-00144). PS was supported by the Center for Functional Nanostructures of the Deutsche Forschungsgemeinschaft within project B2.

\section{A Flux dependence of the ground state energy for large rings with a small non-interacting scattering region}

In this appendix, we discuss the flux dependence of the ground state energy for a ring containing a non-interacting scatterer. The scattering region of length $L_{\mathrm{S}}$ is connected to a disorder-free lead of length $L_{\mathrm{L}}$. This arrangement is closed to a ring of total length $L=L_{\mathrm{S}}+L_{\mathrm{L}}$, as shown in Fig. 1 We present a systematic expansion in powers of $1 / L$ starting from the limit of infinite lead length for the flux-dependent part of the ground state energy. This leads to analytic expressions for the asymptotic values of the persistent current and the charge stiffness, as in Ref. 25]. We extend this theory by calculating the first finitesize corrections to the flux-dependent part of the energy and the charge stiffness. These corrections are important to understand the way in which the asymptotic values are approached when we extrapolate to infinite ring size.

The one-particle eigenenergies of the ring are given by the quantization condition

$$
\operatorname{det}\left(I-M_{\mathrm{L}} M_{\mathrm{S}}\right)=0,
$$

where $M_{\mathrm{S}}$ and $M_{\mathrm{L}}$ are the transfer matrices of the system and the lead, respectively. In the presence of time-reversal symmetry, the transfer matrix of a one-dimensional scatterer can be expressed in terms of three independent angles $\alpha, \theta$ and $\varphi$ :

$$
\begin{aligned}
M_{\mathrm{S}} & =\left(\begin{array}{cc}
1 / t^{*} & r^{*} / t^{*} \\
r / t & 1 / t
\end{array}\right) \\
& =\frac{1}{\sin \varphi}\left(\begin{array}{cc}
e^{i \alpha} / \sin \theta & -i \cot \theta+\cos \varphi \\
i \cot \theta+\cos \varphi & e^{-i \alpha} / \sin \theta
\end{array}\right),
\end{aligned}
$$

where the two components correspond to right and left moving particles while $r$ and $t$ are the reflection and transmission amplitudes, respectively. The angle $\alpha$ is the phaseshift associated with the scattering region. Whenever the right-left symmetry is respected, we can set $\varphi=\pi / 2$, and the expression of $M_{\mathrm{S}}$ simplifies considerably. However, this symmetry requirement is not satisfied for disordered samples. In the general case the transmission amplitude is given by $t=e^{\mathrm{i} \alpha} \sin \theta \sin \varphi$.

The transfer matrix of a lead of length $L_{\mathrm{L}}$ for a state with wave number $k \geq 0$ reads

$$
M_{\mathrm{L}}=\exp (\mathrm{i} \Phi)\left(\begin{array}{cc}
\exp \left(\mathrm{i} k L_{\mathrm{L}}\right) & 0 \\
0 & \exp \left(-\mathrm{i} k L_{\mathrm{L}}\right)
\end{array}\right) \text {. }
$$

Here, we have made use of the fact that the flux can be transformed into a boundary condition which may be prescribed in the lead.

Inserting the transfer matrices (14) and (15), the eigenvalue condition (13) yields

$$
\cos (\Phi)=\frac{1}{|t(k)|} \cos (k L+\delta \alpha(k)) .
$$

Here, we have introduced the phase shift $\delta \alpha=\alpha-k L_{\mathrm{S}}$ of the scattering region relative to a perfect lead of the same length $L_{\mathrm{S}}$. The solution of (16) yields the quantized momenta $k$ of the energy eigenstates in the lead.

Since both, $t$ and $\delta \alpha$ are functions of $k$, it is in general impossible to obtain an analytic solution of (16). However, progress can be made in the asymptotic limit of large $L$, which was worked out by Gogolin and Prokof'ev [25] in their study of the persistent current. We extend their approach to calculate the first finite-size corrections of the charge stiffness. Furthermore, a generalization to arbitrary dispersion relation in the lead allows us to discuss continuum and tight-binding models at the same time.

The eigenvalue condition (16) can be rewritten as

$$
k=k_{n}^{0}+\frac{1}{L} f_{ \pm}(k, \Phi) .
$$

Here, $k_{n}^{0}=2 \pi n / L$ with $n \geq 0$ denotes the eigenvalues in the case of perfect transmission with $|t|=1$ and $\delta \alpha=0$. 
Following the notation of Ref. [25], we have furthermore introduced

$$
f_{ \pm}(k, \Phi)= \pm \operatorname{Arccos}(|t(k)| \cos \Phi)-\delta \alpha(k)
$$

By Arccos, we denote the principal branch of the inverse cosine function that takes values in the interval $[0, \pi]$. In order to ensure a positive value for $k, f_{-}(k, \Phi)$ should not be used for the case $n=0$. The splitting of the solutions of (17) corresponding to "+" and "-" cannot exceed the spacing $2 \pi / L$ between the $k_{n}^{0}$, provided that $\delta \alpha(k)$ is smooth on this scale. This is the case in the limit $L \rightarrow \infty$ and ensures that the order of the solutions with respect to energy is given by $n$.

Iterating (17) and expanding $f_{ \pm}$for large systems, we obtain the expansion

$$
\begin{aligned}
k_{n}^{ \pm}= & k_{n}^{0}+\frac{1}{L} f_{ \pm}\left(k_{n}^{0}, \Phi\right) \\
& +\frac{1}{L^{2}} f_{ \pm}\left(k_{n}^{0}, \Phi\right)\left(\frac{\partial f_{ \pm}(k, \Phi)}{\partial k}\right)_{k=k_{n}^{0}} \\
& +\frac{1}{2 L^{3}} \frac{\partial}{\partial k}\left(f_{ \pm}^{2}(k, \Phi) \frac{\partial f_{ \pm}(k, \Phi)}{\partial k}\right)_{k=k_{n}^{0}}+O\left(\frac{1}{L^{4}}\right)
\end{aligned}
$$

for the solutions of (17) in powers of $1 / L$. Such an expansion is problematic in the vicinity of resonances, when $\mathrm{d} \delta \alpha / \mathrm{d} k$ and $\mathrm{d}|t| / \mathrm{d} k$ are very large. Then, the expansion is valid only for sufficiently large $L$.

We now calculate the ground state energy of the system as a function of the flux to order $1 / L^{2}$. The dispersion relation in the perfect lead will be denoted by $\epsilon(k)$. Using (19), we start by expanding the one-particle energies in powers of $1 / L$ and obtain

$$
\begin{aligned}
\epsilon\left(k_{n}^{ \pm}\right)= & \epsilon\left(k_{n}^{0}\right)+\frac{1}{L}\left(\frac{\partial \epsilon}{\partial k} f_{ \pm}(k, \Phi)\right)_{k=k_{n}^{0}} \\
& +\frac{1}{2 L^{2}} \frac{\partial}{\partial k}\left(\frac{\partial \epsilon}{\partial k} f_{ \pm}^{2}(k, \Phi)\right)_{k=k_{n}^{0}} \\
& +\frac{1}{6 L^{3}} \frac{\partial^{2}}{\partial k^{2}}\left(\frac{\partial \epsilon}{\partial k} f_{ \pm}^{3}(k, \Phi)\right)_{k=k_{n}^{0}}+O\left(\frac{1}{L^{4}}\right)
\end{aligned}
$$

For an odd number $N$ of spinless electrons in the ring, all occupied states $n$ come in pairs $([n,-]$ and $[n,+])$, except for the one corresponding to $n=0$. The total ground state energy then reads

$$
\begin{aligned}
& E_{0}^{\mathrm{odd}}(\Phi)=\epsilon\left(k_{0}^{+}\right)+\sum_{n=1}^{n_{\mathrm{F}}}\left[\epsilon\left(k_{n}^{+}\right)+\epsilon\left(k_{n}^{-}\right)\right] \\
& =\epsilon(0)+\frac{1}{2 L^{2}}\left(\frac{\partial^{2} \epsilon}{\partial k^{2}}[\operatorname{Arccos}(|t| \cos \Phi)-\delta \alpha]^{2}\right)_{k=0} \\
& \quad+\sum_{n=1}^{n_{\mathrm{F}}}\left\{2 \epsilon\left(k_{n}^{0}\right)-\frac{2}{L}\left(\frac{\partial \epsilon}{\partial k}\right)_{k=k_{n}^{0}} \delta \alpha\left(k_{n}^{0}\right)\right. \\
& +\frac{1}{L^{2}} \frac{\partial}{\partial k}\left(\frac{\partial \epsilon}{\partial k}\left[\operatorname{Arcos}^{2}(|t| \cos \Phi)+\delta \alpha^{2}\right]\right)_{k=k_{n}^{0}} \\
& \quad-\frac{1}{3 L^{3}} \frac{\partial^{2}}{\partial k^{2}}\left(\frac{\partial \epsilon}{\partial k}\left[3 \delta \alpha \operatorname{Arccos}^{2}(|t| \cos \Phi)+\delta \alpha^{3}\right]\right)_{k=k_{n}^{0}} \\
& \quad+O\left(\frac{1}{L^{3}}\right) .
\end{aligned}
$$

The sum runs up to $n_{\mathrm{F}}=(N-1) / 2$. We have assumed $(\partial \epsilon / \partial k)_{k=0}=0$ and kept all terms which can give rise to contributions up to order $1 / L^{2}$. The first term in the sum is the ground state energy in the absence of scattering. For finite filling, i.e. for $N$ of order $L$, it is proportional to $L$ while the second term representing the energy change due to the scattering potential is of order 1 . The third and fourth terms are the leading flux-dependent corrections. Since we are interested in the persistent current and the charge stiffness, these are the only terms in the sum which need to be considered further. Converting the sums over $n$ into integrals, these flux-dependent contributions can be expressed as

$$
\begin{aligned}
& \frac{1}{2 \pi L} \int_{\pi / L}^{k_{F}+\pi / L} \mathrm{~d} k \frac{\partial}{\partial k}\left(\frac{\partial \epsilon}{\partial k} \operatorname{Arccos}^{2}(|t| \cos \Phi)\right) \\
&= \frac{\hbar v_{\mathrm{F}}}{2 \pi L} \operatorname{Arccos}{ }^{2}\left(\left|t\left(k_{F}\right)\right| \cos (\Phi)\right) \\
&+\frac{1}{2 L^{2}}\left\{\frac{\partial}{\partial k}\left(\frac{\partial \epsilon}{\partial k} \operatorname{Arccos}^{2}(|t| \cos \Phi)\right)_{k=k_{\mathrm{F}}}\right. \\
&\left.-\left(\frac{\partial^{2} \epsilon}{\partial k^{2}} \operatorname{Arcos}^{2}(|t| \cos \Phi)\right)_{k=0}\right\} \\
&+O\left(\frac{1}{L^{3}}\right)
\end{aligned}
$$


and

$$
\begin{aligned}
-\frac{1}{2 \pi L^{2}} \int_{\pi / L}^{k_{F}+\pi / L} \mathrm{~d} k \frac{\partial^{2}}{\partial k^{2}}\left(\frac{\partial \epsilon}{\partial k} \delta \alpha \operatorname{Arccos}^{2}(|t| \cos \Phi)\right) \\
=-\frac{1}{2 \pi L^{2}}\left\{\frac{\partial}{\partial k}\left(\frac{\partial \epsilon}{\partial k} \delta \alpha \operatorname{Arccos}^{2}(|t| \cos \Phi)\right)_{k=k_{\mathrm{F}}}\right. \\
\left.-\left(\frac{\partial^{2} \epsilon}{\partial k^{2}} \delta \alpha \operatorname{Arcos}^{2}(|t| \cos \Phi)\right)_{k=0}\right\} \\
+O\left(\frac{1}{L^{3}}\right)
\end{aligned}
$$

respectively. Here, $k_{\mathrm{F}}=2 \pi n_{\mathrm{F}} / L$ is the Fermi wave number and $v_{\mathrm{F}}=(\partial \epsilon / \hbar \partial k)_{k=k_{\mathrm{F}}}$ is the Fermi velocity. Taking the derivative of the leading flux-dependent term of the ground state energy

$$
E_{0}^{\mathrm{odd}(1)}(\Phi)=\frac{\hbar v_{\mathrm{F}}}{2 \pi L} \operatorname{Arccos}^{2}\left(\left|t\left(k_{F}\right)\right| \cos (\Phi)\right)
$$

with respect to the flux $\phi$, one obtains the asymptotic form of the persistent current given in (1) for an odd number of particles. The leading order of the charge stiffness of (8) is obtained as

$$
\begin{aligned}
D^{(1)} & =-\frac{L}{2}\left(E_{0}^{\text {odd }(1)}(0)-E_{0}^{\text {odd }(1)}(\pi)\right) \\
& =\frac{\hbar v_{\mathrm{F}}}{2}\left[\frac{\pi}{2}-\operatorname{Arccos}\left(\left|t\left(k_{F}\right)\right|\right)\right] .
\end{aligned}
$$

As we will show below, this last result is independent of the parity of the number of particles.

The first finite size-correction to these asymptotic values follows from the second-order contribution $E_{0}^{(2)}$ to the total energy. Using the terms of order $1 / L^{2}$ from (22) and (23), and taking into account the contribution from the particle in the state $[0,+]$ in the second line of (21), we obtain the correction to the charge stiffness for an odd number $N$ of particles

$$
\begin{aligned}
D^{\text {odd }(2)}= & -\frac{L}{2}\left(E_{0}^{\operatorname{odd}(2)}(0)-E_{0}^{\operatorname{odd}(2)}(\pi)\right) \\
=-\frac{1}{2 L}\{ & \left(\left[\frac{\partial^{2} \epsilon}{\partial k^{2}}(\delta \alpha-\pi)+\hbar v_{\mathrm{F}} \frac{\mathrm{d} \delta \alpha}{\mathrm{d} k}\right]\left(\frac{\pi}{2}-\operatorname{Arccos}(|t|)\right)\right. \\
& \left.+\hbar v_{\mathrm{F}}(\delta \alpha-\pi) \frac{\mathrm{d}|t|}{\mathrm{d} k} \frac{1}{\sqrt{1-|t|^{2}}}\right)_{k=k_{\mathrm{F}}} \\
& \left.+\left(\frac{\partial^{2} \epsilon}{\partial k^{2}} \delta \alpha\left(\frac{\pi}{2}-\operatorname{Arccos}(|t|)\right)\right)_{k=0}\right\} .
\end{aligned}
$$

The last term vanishes if we assume that $|t(k=0)|=0$.

In order to treat also the case of an even number of particles, we subtract the contribution of the particle in the one-body state $\left[n_{\mathrm{F}},+\right]$ from the total energy of Eq.
(21) and obtain

$$
\begin{aligned}
E_{0}^{\text {even }}(\Phi)= & E_{0}^{\text {odd }}(\Phi)-\epsilon\left(k_{n_{\mathrm{F}}}^{+}\right) \\
= & E_{0}^{\text {odd }}(\Phi)-\epsilon\left(k_{\mathrm{F}}\right)-\frac{1}{L}\left(\frac{\partial \epsilon}{\partial k} f_{+}(k, \Phi)\right)_{k=k_{\mathrm{F}}} \\
& -\frac{1}{2 L^{2}} \frac{\partial}{\partial k}\left(\frac{\partial \epsilon}{\partial k} f_{+}^{2}(k, \Phi)\right)_{k=k_{\mathrm{F}}}+O\left(\frac{1}{L^{3}}\right) .
\end{aligned}
$$

With these additional terms, one obtains the leading flux-dependent term of the ground state energy for an even number of particles as

$$
E_{0}^{\text {even }(1)}(\Phi)=\frac{\hbar v_{\mathrm{F}}}{2 \pi L} \operatorname{Arccos}^{2}\left(\left|t\left(k_{F}\right)\right| \cos (\Phi-\pi)\right) .
$$

The derivative with respect to $\phi$ leads to the asymptotic form of the persistent current of Eq. (2) for an even number of particles, and the result for the leading contribution to the charge stiffness agrees with (25).

For the first finite-size correction to the stiffness we obtain

$$
\begin{aligned}
D^{\operatorname{even}(2)}= & \frac{L}{2}\left(E_{0}^{\operatorname{even}(2)}(0)-E_{0}^{\operatorname{even}(2)}(\pi)\right) \\
=-\frac{1}{2 L}\{ & {\left[\frac{\partial^{2} \epsilon}{\partial k^{2}} \delta \alpha+\hbar v_{\mathrm{F}} \frac{\mathrm{d} \delta \alpha}{\mathrm{d} k}\right]\left(\frac{\pi}{2}-\operatorname{Arccos}(|t|)\right) } \\
& \left.+\hbar v_{\mathrm{F}} \delta \alpha \frac{\mathrm{d}|t|}{\mathrm{d} k} \frac{1}{\sqrt{1-|t|^{2}}}\right\}_{k=k_{\mathrm{F}}},
\end{aligned}
$$

which differs from the case of an odd number of particles (26).

¿From Eqs. (26) and (29) one can see that the $1 / L$ scaling for approaching the asymptotic values of the stiffness is problematic close to resonances, where $\mathrm{d} \delta \alpha / \mathrm{d} k$ and $\mathrm{d}|t| / \mathrm{d} k$ are large, and $|t|$ approaches 1 . Assuming an isolated Breit-Wigner resonance [35], the Wigner time is proportional to $g$ and the corrections $D^{(2)}$ are essentially given by the half width of the resonance. Outside resonances where $\delta \alpha \ll 1$ and for small $|t|$, the leading correction to the stiffness can be approximated by

$$
D^{(2)} \approx-\frac{1}{2 L} \hbar v_{\mathrm{F}}|t| \frac{\mathrm{d} \delta \alpha}{\mathrm{d} k} .
$$

Therefore, one obtains for this case

$$
\ln \left(\frac{D}{D_{\infty}}\right) \approx \ln \left(\frac{D^{(1)}+D^{(2)}}{D^{(1)}}\right) \simeq-\frac{1}{L} \frac{\mathrm{d} \delta \alpha}{\mathrm{d} k},
$$

and the Wigner time gives the slope of the scaling curve. The above arguments are valid in the non-interacting case. However, the intuition developed in this case is also useful to interpret our numerical results for the interacting case.

\section{B Conductance of a NSN region from persistent current}

In this appendix we treat the case of a superconductor between two metallic leads. This is a striking example of 
a correlated system exhibiting non-Fermi liquid behavior. It will be demonstrated that the correct result for the conductance can be obtained from the persistent current by means of (3).

\section{B.1 Double Andreev scattering}

The Andreev scattering at a NS junction, i.e. the interface between a normal metal and a superconductor, is a wellknown phenomenon. In an Andreev scattering process, an electron coming from the normal metal is reflected as a hole while a Cooper pair moves on in the superconductor. The linear conductance of the interface between the normal metal and the superconductor in the one-channel case is given by

$$
G=\frac{4 e^{2}}{h} \frac{T}{2-T},
$$

where $T$ is the transmission probability in the normal metal 36, 37. For the normal lead, $T=1$ and one gets that the resistance of a single normal-superconductor interface is the half of the resistance without interface.

In the following, we will consider a NSN double junction consisting of a clean superconducting layer of thickness $L_{\mathrm{S}}$ connected to normal-metal electrodes by perfect interfaces. It is assumed that the superconducting gap $\Delta(x)$ jumps at the interface from zero in the normal metal to its full value $\Delta$ inside the superconductor

$$
\Delta(x)=\Delta \Theta(x) \Theta\left(L_{\mathrm{S}}-x\right)
$$

where $\Theta(x)$ is the step function. This approximation is common in the treatment of mesoscopic superconductors 38. Blonder, Tinkham and Klapwijk 39] calculated the conductance by solving the Bogoliubov-de Gennes equation with this rigid-boundary condition and found for $T=1$ the linear conductance $G=2 e^{2} / h$. This result can be understood by taking two Andreev interfaces with conductance (32) in series.

When we close the two normal metal leads of the NSN junction to a ring, we recover the geometry of the embedding method where the correlated system is formed by the superconductor. It is therefore interesting to see how one can recover the linear conductance from this approach.

\section{B.2 Persistent current and conductance of a superconductor}

For a one-channel ring consisting of a normal conducting region of length $L_{\mathrm{N}}$ and a superconducting region of length $L_{\mathrm{S}}$, the solution of the Bogoliubov-de Gennes equation for a boundary condition analogous to (33) yields the persistent current 40.41

$$
J(\Phi)=\frac{4}{\pi} \frac{e v_{\mathrm{F}}}{L_{\mathrm{N}}+\xi_{0} \tanh \left(L_{\mathrm{S}} / \xi_{0}\right)} \sum_{m=1}^{\infty} \frac{T_{m}(X)}{m} \sin (m \Phi) .
$$

Here, $\xi_{0}=\hbar v_{\mathrm{F}} / \Delta$ is the superconducting coherence length and $T_{m}(X)$ denotes a Chebyshev polynomial in the variable

$$
X=\frac{\cos \left(k_{\mathrm{F}} L\right)}{\cosh \left(L_{\mathrm{S}} / \xi_{0}\right)} .
$$

In the limit $\xi_{0} \rightarrow \infty$, one obtains a normal conducting ring of length $L=L_{\mathrm{N}}+L_{\mathrm{S}}$ with the persistent current

$$
\begin{aligned}
J^{0}(\Phi)=\frac{2}{\pi} \frac{e v_{\mathrm{F}}}{L} \sum_{m=1}^{\infty} \frac{1}{m}[ & \sin \left(m\left(\Phi-k_{\mathrm{F}} L\right)\right) \\
& \left.+\sin \left(m\left(\Phi+k_{\mathrm{F}} L\right)\right)\right] .
\end{aligned}
$$

Apart from a factor of two accounting for the spin, this expression reduces to (1) or (2) for $|t|=1$ depending on the parity of the number of particles per spin. We note, however, that the expression (34) for the NS ring can, in general, not be expressed in the form (1) or (2).

According to (3), the dimensionless conductance $g$ can be obtained from the persistent current at flux $\Phi=\pi / 2$

$$
\begin{aligned}
& J(\pi / 2)=\frac{2}{\pi} \frac{e v_{\mathrm{F}}}{L_{\mathrm{N}}+\xi_{0} \tanh \left(L_{\mathrm{S}} / \xi_{0}\right)} \\
& \times \sum_{m=1}^{\infty} \frac{1}{m}\left\{\sin \left[m\left(\frac{\pi}{2}-\operatorname{Arccos}(X)\right)\right]\right. \\
&\left.+\sin \left[m\left(\frac{\pi}{2}+\operatorname{Arccos}(X)\right)\right]\right\} .
\end{aligned}
$$

By means of the Fourier representation of a sawtooth function, one finds that the absolute value of the persistent current becomes

$$
J(\pi / 2)=\frac{e v_{\mathrm{F}}}{L_{\mathrm{N}}+\xi_{0} \tanh \left(L_{\mathrm{S}} / \xi_{0}\right)} .
$$

In view of this result, the superconducting region can be thought of as a normal-conducting metal of an approximate effective length given by the minimum of $L_{\mathrm{S}}$ and $\xi_{0}$.

It is now straightforward to determine from (38) the dimensionless conductance

$$
\begin{aligned}
g & =\lim _{L_{\mathrm{N}} \rightarrow \infty}\left(\frac{J(\pi / 2)}{J^{0}(\pi / 2)}\right)^{2} \\
& =\lim _{L_{\mathrm{N}} \rightarrow \infty}\left(\frac{L_{\mathrm{N}}+L_{\mathrm{S}}}{L_{\mathrm{N}}+\xi_{0} \tanh \left(L_{\mathrm{S}} / \xi_{0}\right)}\right)^{2} .
\end{aligned}
$$

Here, the persistent current of the normal ring can again be thought of as being obtained from (38) in the limit $\xi_{0} \rightarrow \infty$. We thus recover the correct result $g=1$ for the dimensionless conductance. The leading corrections depend on the ratio $\left[L_{\mathrm{S}}-\xi_{0} \tanh \left(L_{\mathrm{S}} / \xi_{0}\right)\right] / L_{\mathrm{N}}$ between the relative length of the superconductor, i.e. the difference between the real length of the superconducting region and its effective length, and the length of the normal region. Even though here the transmission amplitude remains equal to one in the presence of correlations, this example gives another demonstratation that the embedding methods works, even for having the conductance through a system which is very far to exhibit a Fermi liquid behavior. 


\section{References}

1. Z. Yao, C.L. Kane, C. Dekker, Phys. Rev. Lett. 84, 2941 (2000).

2. R.H.M. Smit, C. Untiedt, G. Rubio-Bollinger, R.C. Segers, J.M. van Ruitenbeek, Phys. Rev. Lett. 91, 076805 (2003).

3. J. Nygård, D.H. Cobden, P.E. Lindelof, Nature 408, 342 (2000).

4. C. Joachim, J.K. Gimzewski, A. Aviram, Nature 408, 541 (2000).

5. see e.g. Special issue on Transport in Molecular Wires, ed. by P. Hänggi, M. Ratner, S. Yaliraki, Chem. Phys. 281, pp. 111-487 (2002).

6. D.L. Maslov, M. Stone, Phys. Rev. B 52, R5539 (1995).

7. I. Safi, H.J. Schulz, Phys. Rev. B 52, R17040 (1995).

8. Y. Meir, N.S. Wingreen, Phys. Rev. Lett. 68, 2512 (1992).

9. S. Datta, Phys. Rev. B 46, 9493 (1992).

10. J. Favand, F. Mila, Eur. Phys. J B 2, 293 (1998).

11. O.P. Sushkov, Phys. Rev. B 64, 155319 (2001).

12. R.A. Molina, D. Weinmann, R.A. Jalabert, G.-L. Ingold, J.-L. Pichard, Phys. Rev. B 67, 235306 (2003).

13. V. Meden, U. Schollwöck, Phys. Rev. B 67, 193303 (2003).

14. V. Meden, S. Andergassen, W. Metzner, U. Schollwöck, K. Schönhammer, Europhys. Lett. 64, 769 (2003).

15. T. Rejec, A. Ramšak, Phys. Rev. B 68, 035342 (2003).

16. T. Rejec, A. Ramšak, Phys. Rev. B 68, 033306 (2003).

17. G. Chiappe, J.A. Vergés, J. Phys.: Condens. Matter 15, 8805 (2003).

18. R. Landauer, IBM J. Res. Dev. 1, 223 (1957).

19. M. Büttiker, Phys. Rev. Lett. 57, 1761 (1986).

20. R. Berkovits, Y. Avishai, Phys. Rev. Lett. 76, 291 (1996).

21. O.P. Sushkov, Phys. Rev. B 67, 195318 (2003).

22. K. Louis, C. Gros, Phys. Rev. B 68, 184424 (2003).

23. C.L. Kane, M.P.A. Fisher, Phys. Rev. Lett. 68, 1220 (1992).

24. O.P. Sushkov, private communication (2003).

25. A.O. Gogolin, N.V. Prokof'ev, Phys. Rev. B 50, 4921 (1994).

26. H.-F. Cheung, Y. Gefen, E.K. Riedel, W.-H. Shih, Phys. Rev. B 37, 6050 (1988).

27. A.J. Leggett, in Granular Nanoelectronics, edited by D.K. Ferry, J.R. Barker, C. Jacoboni, NATO ASI series B, Vol. 251 (Plenum, New York, 1991).

28. E.P. Wigner, Ann. Math. 53, 36 (1951); ibid. 55, 7 (1952).

29. J.-L. Pichard, G. Benenti, G. Katomeris, F. Selva, X. Waintal, in Exotic states in Quantum Nanostructures ed. S. Sarkar, Kluwer, Dordrecht; also available from arXiv:cond-mat/0107380

30. B.L. Altshuler, A.G. Aronov, Solid State Commun. 30, 115 (1979).

31. A.L. Efros, B.I. Shklovskii, J. Phys. C: Solid State Physics 8, L49 (1975).

32. P. Schmitteckert, R.A. Jalabert, D. Weinmann, J.-L. Pichard, Phys. Rev. Lett. 81, 2308 (1998).

33. D. Weinmann, R.A. Jalabert, P. Schmitteckert, J.-L. Pichard, Eur. Phys. J. B 19, 139 (2001).

34. R.A. Molina, D. Weinmann, J.-L. Pichard, in preparation.

35. E.R. Mucciolo, R.A. Jalabert, J.-L. Pichard, J. Phys. I France 7, 1267 (1997).

36. C.W.J. Beenakker, Phys. Rev. B 46, 12841 (1992).

37. C.J. Lambert, J. Phys. Condens. Matter, 3, 6579 (1991).

38. C.W.J. Beenakker, Rev. Mod. Phys. 69, 731 (1997).
39. G.E. Blonder, M. Tinkham, T.M. Klapwijk, Phys. Rev. B 25, 4515 (1982).

40. M. Büttiker, T.M. Klapwijk, Phys. Rev. B 33, 5114 (1986).

41. J. Cayssol, T. Kontos, G. Montambaux, Phys. Rev. B 67, 184508 (2003). 